\title{
Experimental Demonstration of Dual-polarisation NFDM Transmission with $b$-Modulation
}

\author{
Xianhe Yangzhang, Son Thai Le, Vahid Aref, Henning Buelow, Domaniç Lavery and \\ Polina Bayvel, Fellow, IEEE
}

\begin{abstract}
Dual-polarisation Nonlinear Frequency Division Multiplexing (DP-NFDM) transmission has been intensively investigated recently due to its potential of doubling the capacity in comparison to single polarisation NFDM systems. However, up to now, due to many challenges in design and practical implementation, demonstrated data rates of DP-NFDM transmission systems in experiments are still much lower than the record data rate of single polarisation NFDM transmissions (125 $\mathrm{Gb} / \mathrm{s})$. In this work, by employing the concept of b-modulation and developing effective digital signal processing (DSP), we have experimentally demonstrated for the first time a high capacity DP-NFDM transmission system, achieving a net data rate of 220 Gbps with spectral efficiency (SE) of $4 \mathrm{bits} / \mathrm{s} / \mathrm{Hz}$.
\end{abstract}

Index Terms-Non-linear Fourier transform, non-linear frequency-division multiplexing, non-linear transmission scheme.

\section{INTRODUCTION}

$\mathbf{N}$ FDM has been proposed as an potential technique to combat the fibre Kerr non-linearity impairment, which imposes a limitation on the achievable throughput (or SE) of conventional fibre communication systems [1]. The key idea of NFDM transmission is to encode information onto the nonlinear signal spectrum (defined by the Nonlinear Fourier Transform (NFT)) which evolves linearly along the loss-less fibre channel [2]. In this case, the deterministic nonlinear cross-talks among nonlinear spectral components are absent, which potentially leads to higher SE. For the simplicity, the concept of NFDM has been firstly considered for single polarisation transmissions [3]. Many techniques for encoding and transmitting information using the nonlinear spectrum have been investigated [4]-[9]. In addition, many research groups have put a lot of efforts in demonstrating NFDM transmission systems experimentally [10]-[12]. Many encouraging results have been reported, showing that data rates beyond $100 \mathrm{Gbps}$ per channel can be achieved. The highest data rate so far of a single polarisation NFDM transmission is $150 \mathrm{Gbps}$ gross data rate over $976 \mathrm{~km} \mathrm{[4],} \mathrm{which} \mathrm{was} \mathrm{achieved} \mathrm{by}$ modulating simultaneously 222 nonlinear spectral components

Manuscript received October 23rd, 2018; revised month day, 2018.

X. Yangzhang, D. Lavery and P. Bayvel are with the Department of Electrical and Electronic Engineering, University College London, WC1E 7JE London, UK (e-mail: x.yangzhang@ucl.ac.uk). The work of X. Yangzhang is within the COIN project, which has received funding from the European Union's Horizon 2020 research and innovation programme under the MarieSkodowska-Curie grant agreement No.676448. S. T. Le, V. Aref and H. Buelow are with Nokia Bell Labs, 70435 Stuttgart, Germany.

Copyright (c) 2019 IEEE. Personal use of this material is permitted. However, permission to use this material for any other purposes must be obtained from the IEEE by sending a request to pubs-permissions@ieee.org.

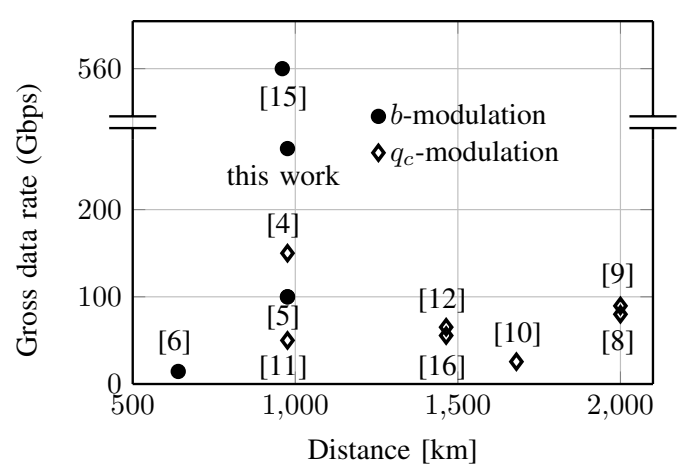

Fig. 1. Gross data rate of recently implemented NFDM systems with $q_{c^{-}}$or $b$-modulation.

(or nonlinear modes). Further increasing the data rate requires increasing the number of modulated nonlinear modes, which has been shown to be challenging due to the nonlinear crosstalks among nonlinear modes in the presence of ASE noise, fibre loss and implementation imperfections [13]. Another effective way to increase the data rate of NFDM transmission is to extend the concept of NFDM to dual polarisation transmission. In recent year, some proof of concept DP-NFDM transmission systems, both in simulation [9] and experiments [10] have been reported. However, high capacity DP-NFDM transmission have not been reported yet. In our previous work [14], we simulated the $b$-modulated DP-NFDM transmission system, and carefully optimised it for high data rate. We showed that the SE of $b$-modulated system achieved 1.2 bits/s/Hz higher SE than $q_{c}$-modulated system. In this work, we experimentally implemented a DP-NFDM transmission system simulated in [14]. By developing efficient DSP, we could successfully modulate 196 nonlinear sub-carriers in each polarisation, achieving a net data rate of $220 \mathrm{Gbps}$ over $976 \mathrm{~km}$ of EDFA-based fibre link. To the best of our knowledge, this is the highest data rate of any NFDM systems in experiment up to date as shown in Fig. 1.

\section{THE DP-NFDM SignAL GENERATION}

In this section, we outline the NFT framework and discuss the DP-NFDM signal generation in brief. In NFDM system, the information is encoded on the non-linear spectra that consist of the continuous spectrum $\left(q_{c i}(\lambda), \lambda \in \mathbb{R}, i=1,2\right)$ and the discrete spectrum (eigenvalues $\lambda_{m} \in \mathbb{C}^{+}$and their spectral amplitude $\left.q_{d i}\left(\lambda_{m}\right), m=1,2,3 \ldots M\right)$. It is also equivalent to 
TABLE I

RECENT RESULTS OF NFDM SYSTEMS.

\begin{tabular}{|c|c|c|c|c|c|c|}
\hline & \multicolumn{2}{|l|}{ Cont. } & \multicolumn{2}{|c|}{ Disc. } & \multirow{2}{*}{ Both } \\
\hline & & $q_{c}$ & $b$ & $q_{d}$ & $b_{k}$ & \\
\hline \multirow{2}{*}{ Sim. } & SP & [8] & & [19] & & [20] \\
\hline & DP & [9] & [15] & [21] & [22] & \\
\hline \multirow{2}{*}{ Exp. } & SP & [4], [7], [11] & [5] & [23], [24] & & [16] \\
\hline & $\overline{\mathrm{DP}}$ & {$[10]$} & [6] & & [25] & [26] \\
\hline
\end{tabular}

encode information on the $\left\{\lambda_{m}, b_{i}(\lambda), b_{m i}\left(\lambda_{m}\right)\right\}$, as they are in one-to-one correspondence to $\left\{\lambda_{m}, q_{c i}(\lambda), q_{d i}\left(\lambda_{m}\right)\right\}[17$, p. 57, Eq. (1.6.19)]. Furthermore, modulating $b$-coefficient allows us to control the time duration of the INFT signal [18]. We categorise some recent implementations of NFDM systems in Tab. I in terms of the type of modulation, singleor dual-polarisation (SP or DP), and simulation or experiment.

The most important property of the non-linear spectra is that over the noiseless and loss-less non-linear Manakov equation, its evolution can be described by simple equations:

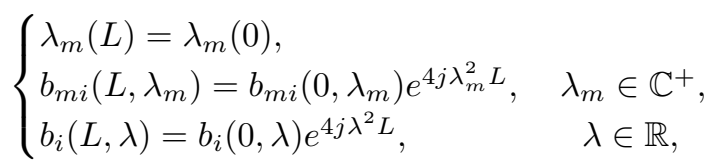

where $L$ is the total distance, $i$ the polarisation index, and $\left\{\lambda_{m}(0), b_{m i}\left(0, \lambda_{m}\right), b_{i}(0, \lambda)\right\}$ the non-linear spectra of the transmitted signal. One can see from the above equations that the spectral amplitude on different non-linear frequency evolves independently during signal propagation in the fibre channel. Therefore, it was proposed in [3] to use an OFDMlike continuous spectrum for NFDM signal generation, as it is written below

$$
u_{i}(\lambda)=\sum_{k=-N_{C} / 2}^{k=N_{C} / 2} c_{k i} \frac{\sin \left(\lambda T_{0}+k \pi\right)}{\lambda T_{0}+k \pi}, \quad i=1,2,
$$

where $T_{0}$ is the NFDM symbol duration, $N_{C}$ is the number of sub-carriers, and $c_{k i}$ is the data on $k$ th sub-carrier on $i$ th polarisation. The discrete spectrum is not used here.

Since the continuous spectrum generates dispersive waves that broaden in the fibre channel, a guard interval (GI) between NFDM symbols in time domain is necessary to avoid intersymbol interference (ISI). The GI insertion is done by zeropadding in the inverse Fourier domain of $\left\{u_{1}(\lambda), u_{2}(\lambda)\right\}$.

To overcome the limiting constraint $\left|b_{1}(\lambda)\right|^{2}+\left|b_{2}(\lambda)\right|^{2}<1$ for $b$-modulation, we apply the following transform $\Gamma_{b}$ on the $\left\{u_{1}(\lambda), u_{2}(\lambda)\right\}$

$$
\begin{aligned}
\Delta & =\sqrt{1-e^{\left(-\left|u_{1}(\lambda)\right|^{2}-\left|u_{2}(\lambda)\right|^{2}\right)}} /\left(\left|u_{1}(\lambda)\right|^{2}+\left|u_{2}(\lambda)\right|^{2}\right) \\
b_{1} & =\Delta \cdot u_{1}(\lambda), \quad b_{2}=\Delta \cdot u_{2}(\lambda) .
\end{aligned}
$$

A detailed discussion about the positive and negative effects of the transform $\Gamma_{b}$ can be found in [14]. The resulted nonlinear spectrum $\left\{b_{1}(\lambda), b_{2}(\lambda)\right\}$ will be used to calculate $a(\lambda)$ and subsequently $\left\{q_{c 1}(\lambda), q_{c 2}(\lambda)\right\}$ via

$$
\begin{aligned}
& a(\lambda)=\sqrt{1-\left|b_{1}(\lambda)\right|^{2}-\left|b_{2}(\lambda)\right|^{2}} e^{j \mathcal{H}\left(\frac{1}{2} \log \left(1-\left|b_{1}(\lambda)\right|^{2}-\left|b_{2}(\lambda)\right|^{2}\right)\right)} \\
& q_{c i}(\lambda)=b_{i}(\lambda) e^{-2 j \lambda^{2} L} / a(\lambda), \quad i=1,2 .
\end{aligned}
$$

Tx DSP

\begin{tabular}{c} 
Symbol mapping \\
IDFT \\
Over-sampling \\
\hline GI insertion \\
\hline DFT \\
\hline$\Gamma_{b}$ \\
\hline PDC \\
\hline DP-INFT \\
QPSK preamble \\
\hline Down-sampling \\
\hline DFT \\
\hline Symbol demapping \\
\hline
\end{tabular}

Fig. 2. Transceiver DSP chain.
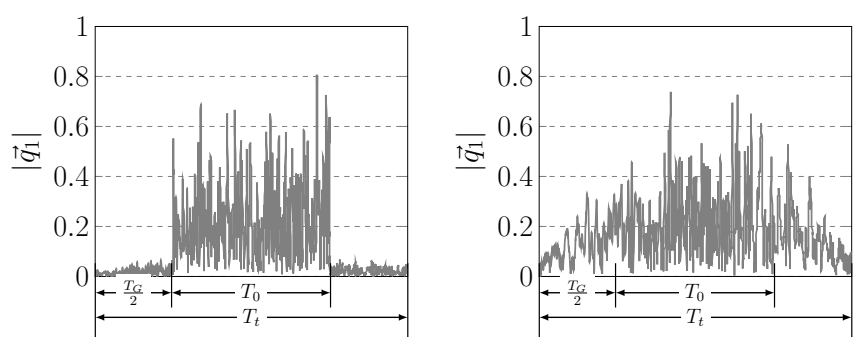

Fig. 3. A realisation of INFT symbols (left) without PDC (right) with PDC.

To reduce the necessary GI to its half, the Pre-Dispersion Compensation (PDC) [27] $\left(e^{-2 j \lambda^{2} L}\right.$ phase-rotation of the nonlinear spectra) is commonly used at the last step of the spectrum generation as in (1). $\left\{q_{c 1}(\lambda), q_{c 2}(\lambda)\right\}$ will be passed to DP-INFT [9, Sec. 3] to generate the time domain signal. We summarise the detailed signal generation and detection steps in white blocks in Fig. 2.

In this work, we transmit DP-NFDM signals of roughly $55 \mathrm{GHz}\left(N_{C} / T_{0}\right)$ linear bandwidth. $c_{k i}$ is randomly drawn from a 32-QAM constellation. The guard interval $T_{G}$ between DP-NFDM symbols is estimated by $T_{G} \approx \pi W \beta_{2} L_{\mathrm{sp}} N_{\mathrm{sp}}=$ $3.57 \mathrm{~ns}$. We set the burst duration $T_{0}=T_{G}$ for simplicity (instead of $T_{0}=5 T_{G}$ for maximum data rate as in [14]). It results in $N_{c}=196$ sub-carriers in the non-linear frequency domain. The system loses half of the SE due to the guard interval. The loss can be reduced by increasing $T_{0}$. We show one of the signal realisations in Fig. 3.

\section{EXPERIMENTAL SETUP AND DSP}

For the transmission experiment, at transmitter, we use four digital-analog converters (DAC) and a polarisation-division multiplexing I/Q modulator to generate the waveform. The output signal is subsequently amplified by an EDFA. Signals are then coupled in and out of the recirculating loop by a 50:50 coupler and travel three loops in our experiment. Each loop has three spans of SMF-28 (Corning) and three EDFAs, making the total distance of the experiment $975.6 \mathrm{~km}$. We chose the same distance as in [4] to allow a direct comparison. 


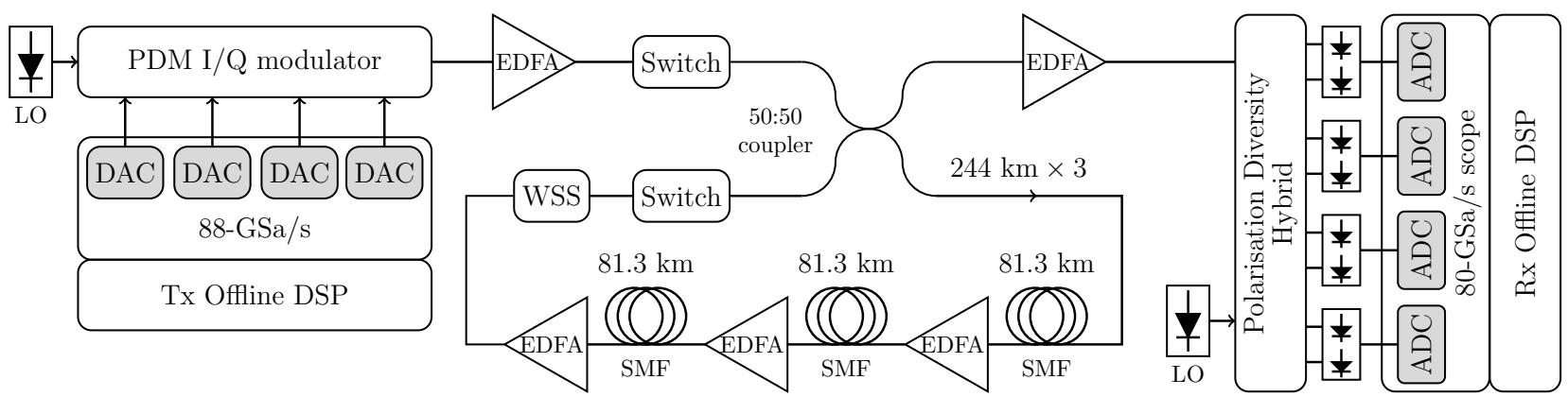

Fig. 4. Recirculating fibre loop experiment setup. The fibre is Corning SMF-28.

As receiver, preceded by another EDFA, an oscilloscope (DSOZ334A) with four channels is used. The sampled data taken by the oscilloscope are saved and processed offline. Two external cavity lasers are used as the optical carrier at the transmitter and as the local oscillator at the receiver. The WSS functions as a optical band-pass filter to remove the out-ofband amplified spontaneous emission noise. Some important parameters of the devices are listed in Tab. II.

TABLE II

SYSTEM PARAMETERS

\begin{tabular}{|c|c|}
\hline fibre type & Corning SMF-28 \\
\hline DSP oversampling rate (Tx/Rx) & 8 \\
\hline EDFA noise figure & $5.5 \mathrm{~dB}$ \\
\hline cavity laser linewidth & $100 \mathrm{kHz}$ \\
\hline DAC Effective number of bits & $4.5 \sim 5$ \\
\hline
\end{tabular}

Several DSP blocks, marked in gray colour in Fig. 2, are added to deal with synchronisation, frequency-offset compensation, arbitrary polarisation rotation and tracking, and phase noise of the laser. In the following, we explain every 'gray' step in the DSP in detail.

1) QPSK preamble: For synchronisation and polarisationdemultiplexing (POL-DEMUX) purposes, single-carrier QPSK signals (55 Gbaud Root-raised cosine pulses with roll-off factor of 0.5) are prepended to the DP-NFDM signals. The QPSK signals occupy $4.3 \%$ of the transmission period. The choice is motivated by the existence of versatile adaptive filters for POL-DEMUX and detection of QPSK signals. The resulted signal in one duty cycle is illustrated in Fig. 5. The NFDM training sequence is to remove any scaling or phase rotation in the non-linear frequency domain.

\begin{tabular}{|c|c|c|}
\hline $\begin{array}{c}\text { QPSK } \\
4.3 \%\end{array}$ & \begin{tabular}{|c|} 
NFDM \\
TS \\
$4.08 \%$
\end{tabular} & $\begin{array}{c}\text { NFDM } \\
\text { Symbols } \\
91.62 \%\end{array}$ \\
\hline
\end{tabular}

Fig. 5. Signal structure in one duty cycle. TS stands for training symbols.

2) Frequency Offset Compensation: The frequency offset compensation was done with the help of the residual carrier.

3) Synchronisation: The QPSK preambles are used for synchronisation. The chromatic dispersion (CD) compensation is first applied to the whole signal. By calculating the crosscorrelation between the amplitude of the known QPSK 'pilot' and the amplitude of the CD-compensated signal, the beginning of the DP-NFDM signal can be easily located.
4) POL-DEMUX: The recursive least square multi-sample per symbol constant-modulus algorithm (RLS-msps-CMA) [28, Sec. V-3)] was performed on the CD-compensated QPSK 'pilot' to estimate the $2 \times 2 \mathrm{MIMO}$ filter that tracks the arbitrary polarisation rotation and compensates the residual ISI. The obtained filter is then applied on the non-compensated DPNFDM signals. Without phase noise compensation, a ring constellation is expected from the RLS-CMA.

5) Phase Noise Compensation: The phase noise is assumed to be perfectly tracked, and constant within one DP-NFDM symbol. The assumption is realised by comparing the phase of the theoretical waveform (from the noiseless simulation) with the received one, then average within one symbol period. It is equivalent to compensate in the non-linear frequency domain as $e^{j \phi} q(t) \leftrightarrow e^{-j \phi}\left\{q_{c 1}(\lambda), q_{c 2}(\lambda)\right\}$.

\section{EXPERIMENTAL RESULTS}

To verify our transceiver DSP chain, we first carried out a back-to-back experiment. The waveforms generated by INFT for each launch power are different. In our back-to-back experiment, however, the transmitted signal power is fixed (set by the EDFA following I/Q modulator). We change only the waveform that is designed for its particular launch power. To collect statistics, we collect at every designed launch power 5 traces of 512 NFDM symbols, each having 196 sub-carriers. The Signal-to-Noise Ratios (SNRs) of the received signal can be calculated from the error vector magnitude (EVM). Fig. 6(a) shows the measured SNR and their average in terms of the designed launch power at the receiver in the back-toback scenario. It can be seen that, when we use the waveforms designed for launch power larger than $-3 \mathrm{dBm}$, the measured SNR starts to decrease. This is attributed to the reduced accuracy of INFT-NFT algorithm at high power, and DAC quantisation noise.

We perform the transmission experiment over 12 spans of $81.3 \mathrm{~km}$ SMF. Waveforms are launched at their designed launch powers, and the polarisation state is also randomly changed at the transmitter. Fig. 6(b) shows the measured SNRs and their average in terms of the launch power. To compute the SE, we estimate the Mutual Information (MI) (of individual sub-carrier detection) by assuming the channel conditional distribution as a Gaussian distribution [29]. The net data rate and SE are computed by

Net rate $=\frac{\mathrm{MI} \cdot N_{c} \cdot 2}{\left(T_{0}+T_{G}\right) \mathrm{ns}}(\mathrm{Gb} / \mathrm{s}), \mathrm{SE}=\frac{\text { Net rate }}{55 \mathrm{GHz}}(\mathrm{bits} / \mathrm{s} / \mathrm{Hz})$. 

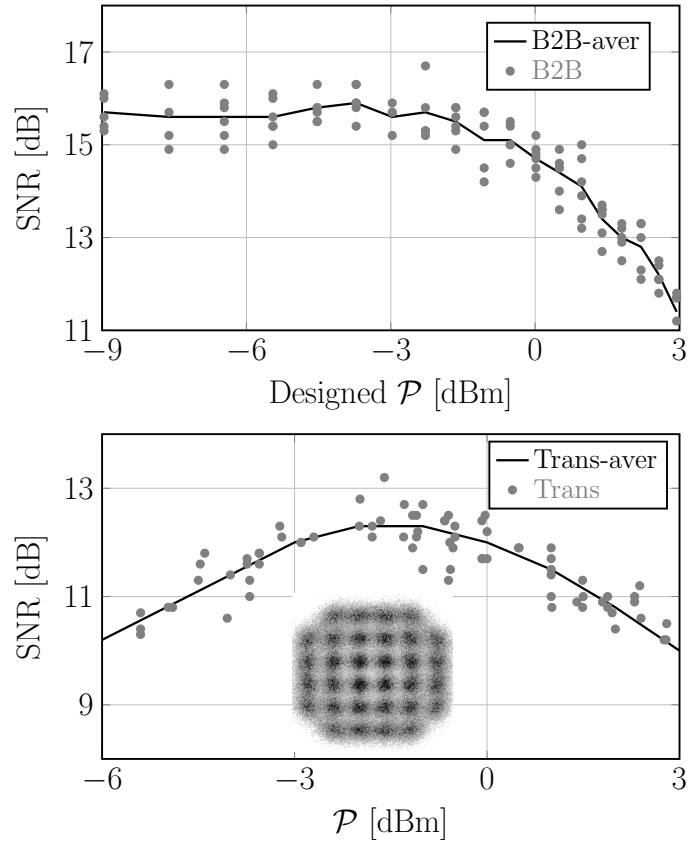

(a)

Fig. 6. Measured SNRs and their average (a) in terms of the designed launch power in the back-to-back scenario, (b) in terms of the launch power over 12 spans of $81.3 \mathrm{~km}$ SMF. Below the curve is one received constellation at signal power of $-1.3 \mathrm{dBm}, \mathrm{Q}$ factor $=3.7 \mathrm{~dB}, \mathrm{SE} \approx 2 \mathrm{bits} / \mathrm{s} / \mathrm{Hz} / \mathrm{pol}$.

This net data rate can be achieved by a soft-decision capacityapproaching binary code such as spatially coupled LDPC codes [30] At the launch power of $-1.3 \mathrm{dBm}$, the system reached its maximum net rate-220Gbps, SE of 4 bits/s/Hz. The corresponding constellation is also plotted in Fig. 6(b).

\section{Conclusions}

In summary, we demonstrate in experiment a $220 \mathrm{Gbps} b$ modulated DP-NFDM system, achieving a SE of $4 \mathrm{bits} / \mathrm{s} / \mathrm{Hz}$. We experimentally verified the system design developed in [15], and added extra DSP blocks to handle frequency offset compensation, synchronisation, POL-DEMUX and phase noise compensation through a QPSK 'pilot' preamble. The data rate can potentially be improved by increasing the number of sub-carrier in each DP-NFDM symbol.

\section{REFERENCES}

[1] R. J. Essiambre, G. Kramer, P. J. Winzer, G. J. Foschini, and B. Goebel, "Capacity limits of optical fiber networks," J. Lightw. Technol., vol. 28, pp. 662-701, Feb 2010.

[2] M. I. Yousefi and F. R. Kschischang, "Information transmission using the nonlinear Fourier transform, Part I: Mathematical tools," IEEE Trans. Inf. Theory, vol. 60, pp. 4312-4328, July 2014.

[3] J. E. Prilepsky, S. A. Derevyanko, and S. K. Turitsyn, "Nonlinear spectral management: Linearization of the lossless fiber channel," Opt. Express, vol. 21, pp. 24344-24367, Oct 2013.

[4] S. T. Le, V. Aref, and H. Buelow, "High speed precompensated nonlinear frequency-division multiplexed transmissions," J. Lightw. Technol., vol. 36, pp. 1296-1303, Mar 2018

[5] S. T. Le, K. Schuh, F. Buchali, and H. Buelow, "100 Gbps b-modulated nonlinear frequency division multiplexed transmission," in Opt. Fiber Commun. Conf. (OFC), (San Diego, CA, USA), W1G.6, Mar 2018.

[6] T. Gui, G. Zhou, C. Lu, A. P. T. Lau, and S. Wahls, "Nonlinear frequency division multiplexing with b-modulation: shifting the energy barrier," Opt. Express, vol. 26, pp. 27978-27990, Oct 2018.
[7] S. Le, V. Aref, and H. Buelow, "Nonlinear signal multiplexing for communication beyond the Kerr nonlinearity limit," Nat. Photon., vol. 11, pp. 570-576, July 2017.

[8] S. Civelli, E. Forestieri, and M. Secondini, "Decision-feedback detection strategy for nonlinear frequency-division multiplexing," Opt. Express, vol. 26, pp. 12057-12071, Apr 2018.

[9] J.-W. Goossens, M. I. Yousefi, Y. Jaoun, and H. Hafermann, "Polarization-division multiplexing based on the nonlinear fourier transform," Opt. Express, vol. 25, pp. 26437-26452, Oct 2017.

[10] W. A. Gemechu, T. Gui, J. Goossens, M. Song, S. Wabnitz, H. Hafermann, A. P. T. Lau, M. I. Yousefi, and Y. Jaoun, "Dual polarization nonlinear frequency division multiplexing transmission," IEEE Photon. Technol. Lett., vol. 30, pp. 1589-1592, Sept 2018.

[11] S. T. Le and H. Buelow, " $64 \times 0.5$ Gbaud Nonlinear Frequency Division Multiplexed Transmissions With High Order Modulation Formats," $J$. Lightwave Technol., vol. 35, pp. 3692-3698, Sept 2017.

[12] S. T. Le, H. Buelow, and V. Aref, "Demonstration of $64 \times 0.5$ Gbaud nonlinear frequency division multiplexed transmission with 32QAM," in Optical Fiber Communication Conference, p. W3J.1, 2017.

[13] X. Yangzhang, D. Lavery, P. Bayvel, and M. Yousefi, "Impact of perturbations on nonlinear frequency-division multiplexing," J. Lightw. Technol., vol. 36, pp. 485-494, Jan 2018.

[14] X. Yangzhang, V. Aref, S. T. Le, H. Buelow, D. Lavery, and P. Bayvel, "Dual-polarisation Non-linear Frequency-division Multiplexed Transmission with b-Modulation," J. Lightw. Technol., vol. Early Access, Mar 2019.

[15] X. Yangzhang, V. Aref, S. T. Le, H. Blow, and P. Bayvel, "400 Gbps Dual-Polarisation Non-Linear Frequency-Division Multiplexed Transmission with $b$-Modulation," in Eur. Conf. Opt. Commun. (ECOC), (Rome, Italy), We4F.1, Sept 2018.

[16] V. Aref, S. T. Le, and H. Buelow, "Modulation over nonlinear fourier spectrum: Continuous and discrete spectrum," J. Lightw. Technol., vol. 36, pp. 1289-1295, March 2018.

[17] M. Ablowitz and H. Segur, Solitons and the Inverse Scattering Transform. SIAM studies in applied and numerical mathematics: Society for Industrial and Applied Mathematics, SIAM, 1981.

[18] S. Wahls, "Generation of time-limited signals in the nonlinear fourier domain via b-modulation," in Eur. Conf. Opt. Commun. (ECOC), (Gothenburg, Sweden), W.3.C.6, Sept 2017.

[19] S. Hari, M. I. Yousefi, and F. R. Kschischang, "Multieigenvalue communication," J. Lightwave Technol., vol. 34, pp. 3110-3117, Jul 2016.

[20] I. Tavakkolnia and M. Safari, "Signalling over nonlinear fibre-optic channels by utilizing both solitonic and radiative spectra," in Eur. Conf. on Networks and Commun. (EuCNC), pp. 103-107, June 2015.

[21] A. Span, V. Aref, H. Bülow, and S. ten Brink, "Precoding for dual polarization soliton transmission," arXiv:1808.09904, Aug 2018.

[22] T. Gui, T. H. Chan, C. Lu, A. P. T. Lau, and P.-K. A. Wai, "Alternative decoding methods for optical communications based on nonlinear fourier transform," J. Lightwave Technol., vol. 35, pp. 1542-1550, May 2017.

[23] Z. Dong, S. Hari, T. Gui, K. Zhong, M. I. Yousefi, C. Lu, P. A. Wai, F. R. Kschischang, and A. P. T. Lau, "Nonlinear Frequency Division Multiplexed Transmissions Based on NFT," IEEE Photon. Technol. Lett., vol. 27, pp. 1621-1623, Aug 2015.

[24] H. Buelow, V. Aref, and W. Idler, "Transmission of waveforms determined by 7 eigenvalues with psk-modulated spectral amplitudes," in Eur. Conf. Opt. Commun. (ECOC), Tu.3.E.2, Sep. 2016.

[25] S. Gaiarin, A. M. Perego, E. P. da Silva, F. D. Ros, and D. Zibar, "Dualpolarization nonlinear fourier transform-based optical communication system," Optica, vol. 5, pp. 263-270, Mar 2018.

[26] F. Da Ros, S. Civelli, S. Gaiarin, E. Porto da Silva, N. De Renzis, M. Secondini, and D. Zibar, "Dual-polarization NFDM transmission with continuous and discrete spectral modulation," J. Lightw. Technol., vol. Early Access, Mar 2019.

[27] I. Tavakkolnia and M. Safari, "Dispersion pre-compensation for NFTbased optical fiber communication systems," in Conf. on Lasers and Electro-Optics (CLEO), (San Jose, CA, USA), SM4F.4, Jun 2016.

[28] M. S. Faruk and S. J. Savory, "Digital Signal Processing for Coherent Transceivers Employing Multilevel Formats," J. Lightw. Technol., vol. 35, pp. 1125-1141, March 2017.

[29] A. Alvarado, T. Fehenberger, B. Chen, and F. M. J. Willems, "Achievable information rates for fiber optics: Applications and computations," $J$. Lightw. Technol., vol. 36, pp. 424-439, Jan 2018.

[30] L. Schmalen, V. Aref, J. Cho, D. Suikat, D. Rösener, and A. Leven, "Spatially Coupled Soft-Decision Error Correction for Future Lightwave Systems," J. Lightw. Technol., vol. 33, pp. 1109-1116, Mar 2015. 